\title{
THE IMPORTANCE OF COMPENSATING VASOCONSTRICTION IN UNANESTHETIZED AREAS IN THE MAINTENANCE OF BLOOD PRESSURE DURING SPINAL ANESTHESIA ${ }^{1}$
}

\author{
By C. NEUMANN, A. D. FOSTER, JR., AND E. A. ROVENSTINE \\ (From the Hospital of the Rockefeller Institute for Medical Research, and the Division of \\ Anesthesia, Bellevue Hospital, New York City)
}

(Received for publication September 25, 1944)

The trauma of surgery or injury is regularly associated with alterations in the circulatory status of the individual. Such alterations may be limited to the site of injury, but if the injury is at all extensive, and especially if associated with hemorrhage and severe pain, a more generalized circulatory response may be expected. This response may involve change in cardiac output, volume of blood, constitution of blood, volume of extracellular fluid, circulation time, blood pressure, and peripheral circulation (1). When the response is inadequate, various types of circulatory insufficiency, such as peripheral vascular collapse or even shock, may supervene. Measurement of the response while it is proceeding is important in order that therapeutic measures may be employed as early as needed. Clinically, observation of the blood pressure is usually the sole quantitative criterion of the changing circulatory status, although attention is also paid to quality and rate of the pulse, the color and moisture of the skin, and depth and rate of respiration. The other constituents of the circulatory response to trauma are subject to measurement in human beings, but are usually neglected because they are not readily and conveniently ascertainable.

In the course of a study of the circulatory adjustments attendant upon surgical trauma, plethysmographic registration of the peripheral circulation has been utilized as an additional clinical guide to the circulatory response. This was undertaken in the belief that efforts to maintain blood volume, blood pressure, and cardiac output are directed toward the continuation of an adequate supply of blood to the tissues of the body. If the peripheral cutaneous circulation can be maintained, then, presumably, the circulatory adjustments are adequate to the needs of the more

1 This is the 14th paper reporting the results of studies of the small blood vessels and related subjects. important organs. It has been necessary to evaluate the effects of various types of anesthesia upon the peripheral circulation in view of the universal use of anesthesia in the course of major surgical operations. This report deals with the study of certain peripheral vascular adjustments during spinal and regional anesthesia.

\section{APPARATUS AND METHODS}

The pneumoplethysmograph (2) was used to record the rhythmic variations in the volume of the fingers and toes dependent upon constriction and dilatation of the small peripheral blood vessels (3). Such rhythmic pulsations are recorded as pulse waves synchronous with the cardiac beat and alpha waves. The latter occur irregularly at a rate 4 to 7 times per minute and vary in size up to 10 times that of pulse waves. The pulse raves may be as small as 1 to $2 \mathrm{c}$. $\mathrm{mm}$. per $5 \mathrm{ml}$. of finger or toe or as large as $20 \mathrm{c}$. $\mathrm{mm}$. Commonly, large alpha waves occur in association with varying, medium-sized, pulse waves ( 5 to $15 \mathrm{c}$. $\mathrm{mm}$.) and small alpha waves. with very small or very large pulse waves (4).

Twenty-eight patients, ranging in age from 30 to 61 years, were subjected to spinal anesthesia for such surgical procedures as hemorrhoidectomy or herniorrhaphy. The observations reported here were made before the onset of surgical manipulation. The patients had no known hypertension or cardiac or peripheral vascular disease and were regarded as in good health except for their relatively minor surgical complaint. Twenty-four were male and 4 female. The spinal anesthetic agents employed were procaine hydrochloride (50 to $100 \mathrm{mgm}$.) or Monocaine Formate (75 to $150 \mathrm{mgm}$.). The intrathecal injection was made through the third or fourth lumbar intervertebral space while the patients were in the lateral position. For 2 of the patients, a malleable spinal puncture needle was inserted, and the patients then returned to a supine position, after which the anesthetic agent was injected. For the others, a regular spinal puncture needle was inserted, the anesthetic drug injected, the needle withdrawn, and the patient then returned to the supine position.

Plethysmograms were obtained from the index finger and second toe of the same side of the body. They were made with the patients supine and the fingers and toes were within 6 inches of the level of the heart. Recordings were made before the insertion of the spinal puncture needle and after the injection of the anesthetic drug. 
TABLE I

Comparison of the volume of pulse waves of the fingers and toes before and after spinal anesthesia unassociated with decrease in blood pressure

\begin{tabular}{|c|c|c|c|c|c|c|c|c|c|c|c|}
\hline \multirow{2}{*}{ Number } & \multirow{2}{*}{ Age } & \multirow{2}{*}{ Sex } & \multicolumn{2}{|c|}{ Blood pressure } & \multicolumn{2}{|c|}{ Pulse rate } & \multicolumn{2}{|c|}{ Pulse waves-toe } & \multicolumn{2}{|c|}{ Pulse waves-finger } & \multirow{2}{*}{$\begin{array}{l}\text { Upper } \\
\text { limit of } \\
\text { anesthesia }\end{array}$} \\
\hline & & & $\begin{array}{c}\text { Before } \\
\text { anesthesia }\end{array}$ & $\begin{array}{c}\text { After } \\
\text { anesthesia }\end{array}$ & $\begin{array}{c}\text { Before } \\
\text { anesthesia }\end{array}$ & $\begin{array}{c}\text { After } \\
\text { anesthesia }\end{array}$ & $\begin{array}{c}\text { Before } \\
\text { anesthesia }\end{array}$ & $\begin{array}{c}\text { After } \\
\text { anesthesia }\end{array}$ & $\begin{array}{c}\text { Before } \\
\text { anesthesia }\end{array}$ & $\begin{array}{c}\text { After } \\
\text { anesthesia }\end{array}$ & \\
\hline $\begin{array}{r}1 \\
2 \\
3 \\
4 \\
5 \\
6 \\
7 \\
8 \\
9 \\
10 \\
11 \\
12 \\
13 \\
14 \\
15 \\
16 \\
17 \\
18 \\
19 \\
20\end{array}$ & $\begin{array}{c}\text { years } \\
51 \\
38 \\
50 \\
36 \\
47 \\
60 \\
55 \\
50 \\
45 \\
32 \\
47 \\
45 \\
38 \\
30 \\
61 \\
49 \\
50 \\
39 \\
44 \\
64\end{array}$ & $\begin{array}{l}\mathbf{M} \\
\mathbf{M} \\
\mathbf{M} \\
\mathbf{F} \\
\mathbf{M} \\
\mathbf{M} \\
\mathbf{M} \\
\mathbf{M} \\
\mathbf{M} \\
\mathbf{M} \\
\mathbf{M} \\
\mathbf{M} \\
\mathbf{M} \\
\mathbf{F} \\
\mathbf{M} \\
\mathbf{F} \\
\mathbf{M} \\
\mathbf{M} \\
\mathbf{M} \\
\mathbf{M}\end{array}$ & $\begin{array}{l}m m . H g \\
136 / 84 \\
106 / 74 \\
116 / 86 \\
110 / 68 \\
118 / 60 \\
140 / 100 \\
100 / 70 \\
146 / 90 \\
130 / 96 \\
100 / 50 \\
106 / 70 \\
144 / 80 \\
124 / 72 \\
118 / 72 \\
100 / 70 \\
130 / 80 \\
130 / 78 \\
160 / 80 \\
100 / 50 \\
110 / 80\end{array}$ & $\begin{array}{r}m m . H g \\
138 / 80 \\
114 / 50 \\
90 / 70 \\
100 / 50 \\
110 / 74 \\
130 / 90 \\
106 / 78 \\
130 / 80 \\
130 / 96 \\
96 / 60 \\
102 / 60 \\
140 / 78 \\
126 / 70 \\
108 / 80 \\
100 / 60 \\
140 / 80 \\
110 / 80 \\
156 / 76 \\
110 / 60 \\
122 / 78\end{array}$ & $\begin{array}{l}60 \\
58 \\
64 \\
78 \\
76 \\
86 \\
60 \\
84 \\
84 \\
70 \\
96 \\
70 \\
76 \\
84 \\
84 \\
84 \\
90 \\
92 \\
72 \\
74\end{array}$ & $\begin{array}{r}70 \\
66 \\
64 \\
88 \\
66 \\
88 \\
64 \\
86 \\
116 \\
60 \\
86 \\
96 \\
80 \\
80 \\
88 \\
94 \\
92 \\
120 \\
70 \\
66\end{array}$ & $\begin{array}{l}c . m m . \\
4.0 \\
2.0 \\
1.5 \\
4.0 \\
1.5 \\
2.0 \\
8.0 \\
6.0 \\
1.5 \\
1.5 \\
1.0 \\
2.5 \\
2.0 \\
1.5 \\
4.0 \\
4.0 \\
3.0 \\
2.0 \\
8.0 \\
2.0\end{array}$ & \begin{tabular}{|r} 
per $5 l$. \\
13.0 \\
14.0 \\
11.0 \\
15.0 \\
7.0 \\
10.0 \\
15.0 \\
12.0 \\
5.0 \\
12.0 \\
5.0 \\
6.0 \\
8.5 \\
8.0 \\
9.5 \\
10.5 \\
11.0 \\
5.0 \\
14.0 \\
3.5
\end{tabular} & $\begin{array}{c}c . m m . \\
7.5 \\
7.0 \\
3.0 \\
8.0 \\
5.0 \\
8.0 \\
10.0 \\
6.0 \\
3.0 \\
4.0 \\
3.5 \\
5.5 \\
2.0 \\
6.0 \\
4.5 \\
7.0 \\
4.5 \\
6.0 \\
8.0 \\
2.5\end{array}$ & \begin{tabular}{|r} 
per $5 \mathrm{ml}$. \\
3.0 \\
3.0 \\
1.5 \\
5.0 \\
4.0 \\
5.0 \\
3.0 \\
4.0 \\
1.0 \\
1.5 \\
2.0 \\
1.5 \\
1.0 \\
3.5 \\
1.5 \\
4.0 \\
3.0 \\
2.5 \\
5.0 \\
1.5
\end{tabular} & $\begin{array}{l}\mathrm{T}-8 \\
\mathrm{~T}-11 \\
\mathrm{~T}-8 \\
\mathrm{~T}-5 \\
\mathrm{~T}-11 \\
\mathrm{~T}-6 \\
\mathrm{~T}-4 \\
\mathrm{~T}-8 \\
\mathrm{~T}-8 \\
\mathrm{~T}-8 \\
\mathrm{~T}-6 \\
\mathrm{~T}-4 \\
\mathrm{~T}-9 \\
\mathrm{~T}-9 \\
\mathrm{~T}-8 \\
\mathrm{~T}-11 \\
\mathrm{~T}-9 \\
\mathrm{~T}-6 \\
\mathrm{~T}-9 \\
\mathrm{~T}-11\end{array}$ \\
\hline Average & 47 & & $121 / 76$ & $118 / 73$ & 77 & 82 & 3.1 & 9.8 & 5.6 & 2.8 & \\
\hline
\end{tabular}

Measurements of blood pressure were made at 2- to 4-minute intervals by the use of an ordinary sphygmomanometer. Values for pulse rates were taken from the plethysmographic records. All of the studies were made in an environmental temperature within $5^{\circ}$ of $75^{\circ} \mathrm{F}$. Ephedrine sulfate (25 $\mathrm{mgm}$.) was given intravenously to 8 of the patients in whom a progressive fall in blood pressure had occurred following the onset of spinal anesthesia.

\section{RESULTS}

The plethysmograms of fingers and toes obtained before the insertion of the spinal needle showed medium-sized pulse waves and large alpha waves in 19 of the subjects. Such records are suggestive of a state of moderate anxiety (4), not unexpected in patients about to be operated upon. In 9 patients, both the pulse waves and alpha waves were small, indicating generalized peripheral vasoconstriction associated with more intense anxiety. Plethysmograms recorded after injection of the drug and return of the patient to the supine position, but before the inception of anesthesia, showed the same pulse wave-alpha wave pattern as before the insertion of the spinal needle. Thus, at the time of onset of anesthesia, all of the subjects had plethysmographic tracings indicative of moderate to relatively intense vasoconstriction in the fingers and toes.

During the course of spinal anesthesia, 20 patients exhibited no more than a slight change in systolic or diastolic blood pressure and in only 3 of these was there a change in pulse rate greater than 12 beats per minute. The upper level of sensory anesthesia in these patients ascended at least as high as the eleventh but not higher than the fourth thoracic dermatome. The plethysmographic records obtained from the fingers and toes of this group showed relatively consistent alterations in both the pulse and alpha waves. Within 2 to 5 minutes after injection of the drug, the pulse waves in the toe began to increase and within another few minutes attained great magnitude (Table I). The change in volume of the pulse waves was from an average value of $3.1 \mathrm{c} . \mathrm{mm}^{2}$ to an average value of $9.8 \mathrm{c}$. mm. Concurrently, the alpha waves were suppressed and the third simultaneous event was a marked increase in the total volume of the toe tip. In 1 subject, the volume change was as much as 200 c. mm. equalling $1 / 25$ of the total volume of the toe tip (Figure 1).

2 All changes in volume are reported in c. $\mathrm{mm}$. per 5 ml. of finger or toe tip. 
Occurring simultaneously with the changes noted in the toes, were alterations in the pulse wave-alpha patterns in the finger tips. In none of these patients did the level of spinal anesthesia ascend high enough to make it possible that the changes in the finger could be attributed to a direct effect of the anesthetic drug upon the sympathetic fibers passing from the spinal cord to the finger. The changes in the records of the fingers included: (1) a progressive decrease in the volume of the pulse waves from an average of 5.6 c. $\mathrm{mm}$. to $2.8 \mathrm{c}$. $\mathrm{mm}$.; (2) suppression of alpha waves; and (3) a gradual decrease in the total volume of the finger tip of a magnitude of about one third of the simultaneous increase in the total volume of the toe.

In 8 patients following the onset of anesthesia, a fall of systolic pressure of 40 to $80 \mathrm{~mm}$. $\mathrm{Hg}$ occurred and was accompanied by a fall of about one half of this amount in the diastolic. In 4 of these patients (upper limit of sensory anesthesia $\mathrm{T} 8, \mathrm{~T} 4, \mathrm{~T} 4, \mathrm{~T} 4)$, the response of the toes was the same as that seen in the 20 subjects whose arterial tension remained unchanged; the response in the fingers differed in that instead of showin: a decrease in size of pulse waves, there occurred an increase in pulse waves at the time of the fall in blood pressure. When ephedrine sulfate was administered intravenously, the pulse waves of the finger decreased in amplitude, thus re-establishing the pattern of vasoconstriction and restoring the blood pressure to or above the initial level. The vasodilatation of the toe persisted unchanged. In the remaining 4 patients who experienced hypotension (upper limit of sensory anesthesia T7, T6, T4, T3), both toes and fingers showed a marked decrease in the size of the pulse waves. In these, the intravenous administration of ephedrine prompted a large increase in size of pulse waves of the toe and a lesser increase in size of pulse waves of the finger as blood pressure was restored. The expected vasodilatation of the toes during spinal anesthesia thus was not manifest until after the restoration of blood pressure.

\section{DISCUSSION}

The dilatation of cutaneous blood vessels of the lower extremities is a generally accepted counterpart of spinal anesthesia and is attributed to pharmacologic interruption of the sympathetic nerves carrying vasoconstrictor impulses to these vessels (5). Our findings are in close agreement with this concept. The progressive increase in size of the pulse waves can be accounted for

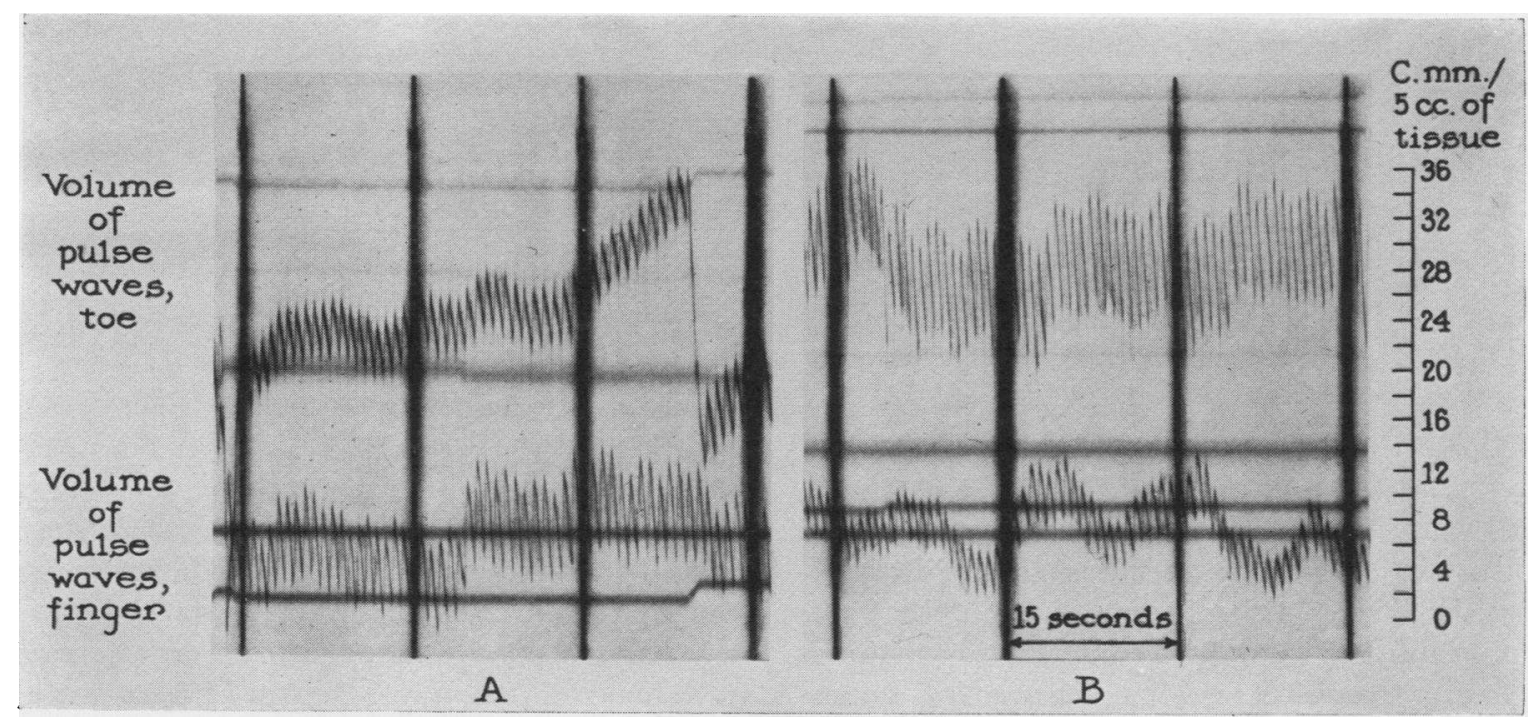

Fig. 1. A Portion of the Original Plethysmographic Record of Patient No. 16 is Shown

A represents the pulsations in the second toe tip and index finger tip before spinal anesthesia. $B$ was recorded 3 minutes after introduction of the anesthetic drug. The pulse waves of the toe increase from 4 to 10.5 c. mm., while those of the finger decrease from 7 to $4 \mathrm{c} . \mathrm{mm}$. 


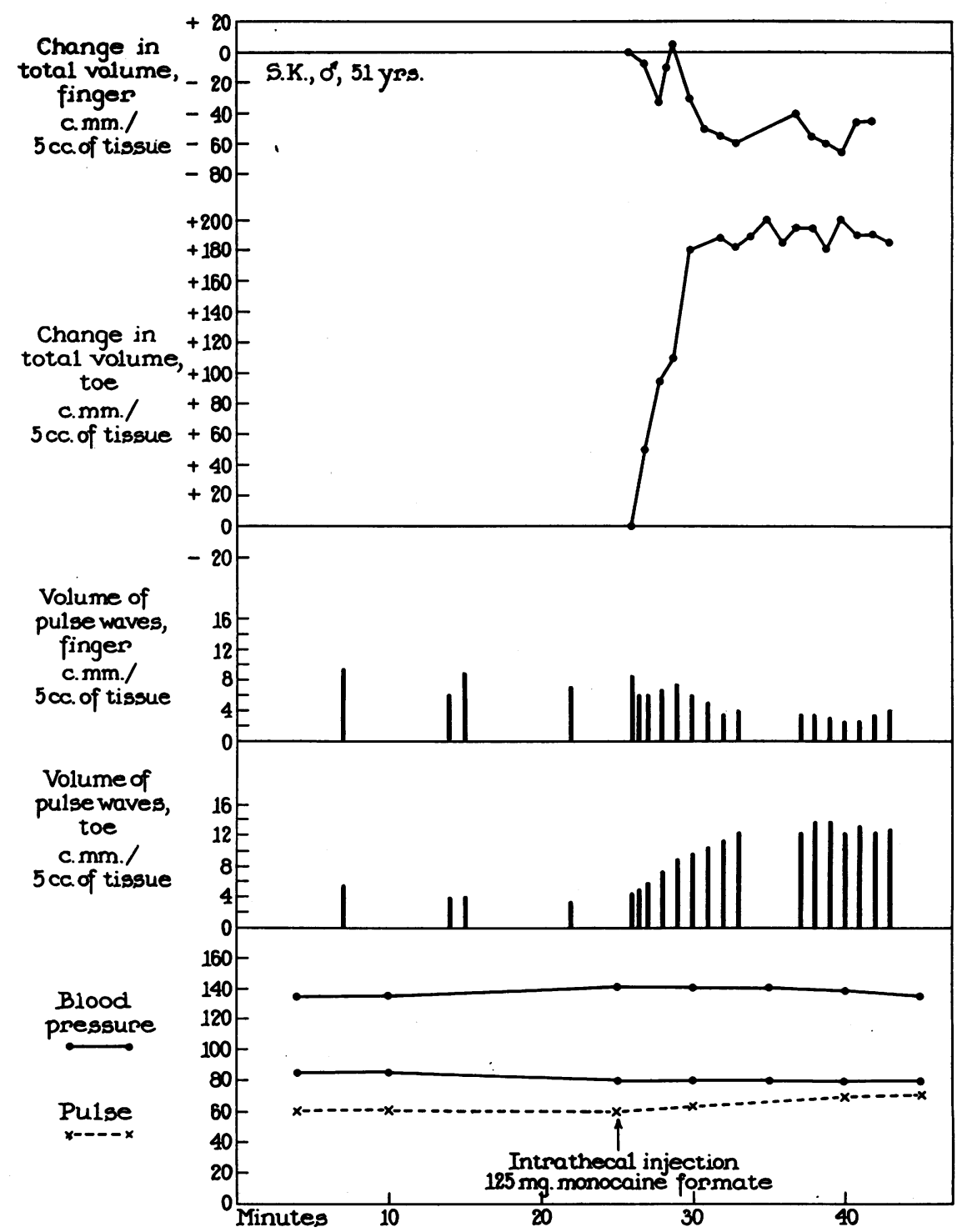

Fig. 2. The Chart Shows: (1) Changes in Total Volume of Finger and Toe, and (2) Changes in Volume of Pulse Waves of Finger and Toe During Onset of Spinal Anesthesia in Patient No. 1

Blood pressure and pulse rate remain essentially unchanged. The upper limit of sensory anesthesia reached $\mathrm{T} 8$.

on the basis of loss of cutaneous arteriolar tone when the sympathetic nervous supply is interrupted. The decrease in size of the alpha waves is explained by postulating interruption of intermittent vasoconstrictor stimuli traversing sympathetic nerves. The production of alpha waves is not fully explainable but the present belief is that in the normal subject (in the absence of anesthesia), at irregular intervals of 8 to 15 seconds, vasoconstrictor impulses traversing the sympathetic nerves reach the small blood vessels and lead to two effects, namely, temporary decrease in volume of pulse waves and temporary decrease in volume of the finger or toe (alpha wave). Intermittent impulses effect intermittent vasoconstriction; passive vasodilatation follows and the resultant pattern is one of rhythmic decrease and increase in the size of the part. The great 
increase in the total volume of the toe during anesthesia may be accounted for by assuming either that the content of the peripheral vascular bed has increased, or that fluid has passed into the local tissues, or that both have occurred.

The changes which were observed in the fingers during the onset and course of spinal anesthesia may be considered as the opposite of those manifest in the toes. Superficially, there may be cause for disagreement with this statement because the alpha waves decreased in volume in both fingers and toes. In the case of the toes, the explanation lay in an interruption of the intermittent impulses necessary for rhythmic vasoconstriction. In the case of the fingers, the evidence supplied by the onset and persistence of small pulse waves suggests that vasoconstrictor impulses have become so closely spaced as to provide no periodic relaxation of the small blood vessels necessary for the formation of alpha waves.

The compensatory occurrence of vasoconstriction in a distant portion may help explain the ability of the body to maintain a normal blood pressure in spite of the marked vasodilatation of a major portion of the peripheral vascular bed during spinal anesthesia. Only in the patients in whom vasoconstriction of the finger occurred and persisted during spinal anesthesia was blood pressure not significantly lowered. When, for reasons not yet understood, this physiologic adjustment did not occur, hypotension ensued.

There are no data in the present experiments to support the theory that vasoconstriction or vasodilatation of such a small peripheral portion as the toe is actually representative of vasoconstriction or dilatation of the entire lower extremity and lower abdominal viscera. If this were not so, however, it would seem unlikely that enough change in circulatory status could result from dilatation of cutaneous vessels of the legs alone to: (1) make the patient during spinal anesthesia highly susceptible to hemorrhagic shock (6); and (2) lead to vasoconstriction of the small vessels of the finger. Similarly, if vasoconstriction or dilatation of the fingers were not representative of vasoconstriction or dilatation of the upper extremity and upper trunk, it would seem unlikely that vasoconstriction of the finger would so uniformly be present when normal blood pressure is retained during spinal anesthesia and be absent when hypotension supervenes. The vasoconstriction of the finger during spinal anesthesia is, then, considered to be: (1) representative of vasoconstriction in a more extensive vascular bed, and (2) compensatory for the vasodilatation of an extensive vascular bed including the lower abdominal viscera and lower extremity (7). The exact extent of these larger vascular beds cannot be defined at present.

The mechanisms of the mediation of the vasoconstrictor response of the vascular bed represented by the finger during spinal anesthesia are presumably none other than those which normally operate to support the blood pressure during physiological stress (7). In the special situation occasioned by spinal anesthesia, the peripheral vascular bed of the lower extremities cannot be called into play, and as a consequence, there is a heightened response of those vascular beds whose sympathetic nerve supply is still intact. Therefore, resiliency of circulatory response during trauma and hemorrhage is lost to the patient having spinal anesthesia, not only because the blood vessels of the lower portion of the body are pharmacologically denervated and thus unable to react, but also because those of the upper portion are already in a state of compensatory partial constriction. Thus, when further demands are made upon the circulation, these vessels undergo very little additional constriction.

The results of these studies of peripheral circulatory response to spinal anesthesia suggest that there may be two, more or less separate, mechanisms which produce hypotension. In one group of patients, the hypotension seems to result from a loss of, or failure of development of, a compensatory constriction of the small blood vessels whose sympathetic nerve supply is intact. In these patients, the administration of ephedrine restored the constriction in the intact vessels but left the vessels of the denervated toes unaffected. This is in keeping with the findings of other investigators who demonstrated that ephedrine acts to constrict only those vessels whose sympathetic nervous supply is intact (8). In the patients who showed decrease in size of pulsations of both toes and fingers concomitant with fall in blood pressure, it is probable that a decrease in cardiac output is in part responsible for the hypotension 
(6). With complete interruption of the sympathetic nervous supply to the lower extremity a decrease in size of pulse waves of the toes cannot indicate vasoconstriction but is most likely a passive reflection of a decrease in cardiac output. The mechanism responsible for such a diminution of cardiac output cannot be explained for the present. It is probable that paralysis of the cardiac nerves is not of primary importance in these patients since in two of them the upper limit of anesthesia was at the sixth and seventh thoracic dermatomes. Again the effects of ephedrine in these patients are consistent with those reported by most investigators who noted a marked increase in cardiac output attendant upon the administration of the drug (9).

There is other evidence which supports the supposition that under many normal conditions there is partial constriction of the peripheral vascular bed, the loss of which in one portion of the body is compensated by additional constriction in another position. In patients in whom the stellate ganglion was anesthetized by infiltration of $11 / 2$ per cent procaine hydrochloride, plethysmograms were taken simultaneously from the index finger tips of the ipsilateral and contralateral hands (10). The plethysmogram from the ipsilateral finger showed vasodilatation and increase in size of the part while the contralateral finger showed vasoconstriction and decrease in total volume. Conversely, the opposite effect occurs when excessive vasoconstriction is induced in one extremity. Faradic stimulation was applied to the distal portion of the lumbar sympathetic chain after section at the level of the first lumbar ganglion during the course of sympathectomy for the reduction of arterial hypertension. The plethysmogram from the ipsilateral toe showed intense vasoconstriction 3 to 5 seconds after the application of the stimulus and of 30 to 45 seconds' duration. The plethysmogram of the index finger showed vasodilatation synchronous with the vasoconstriction of the toe (11).

Three patients subjected to section of the lumbar and splanchnic sympathetic nerves were examined immediately following operation and as long as 2 years later (11). On each occasion, there was marked vasodilatation of the toes and marked vasoconstriction of the fingers. The vasoconstriction of the fingers was far more in- tense than that seen at any time before the operation. None of these patients had a good therapeutic response to sympathectomy. Just as in the normal patient under spinal anesthesia, vasoconstriction in the upper extremity is useful in maintaining a normal blood pressure, so it would seem that vasoconstriction of the upper extremity following lumbar and splanchnic sympathectomy may militate against the desired reduction of blood pressure.

\section{SUMMARY}

Simultaneous plethysmographic records were obtained from the fingers and toes of 28 patients during the onset and early course of spinal anesthesia. In 20 patients who experienced no fall in blood pressure, the expected vasodilatation of the toes occurred, associated with concomitant vasoconstriction of the fingers which was interpreted as representing a compensating mechanism for the support of the circulation. In 4 of the 8 patients who experienced a fall in blood pressure, vasodilatation of the toes occurred unaccompanied by vasoconstriction of the fingers. Following administration of ephedrine, vasoconstriction developed in the fingers and the blood pressure rose, while the vasodilatation of the toes continued. In the other 4, fall in blood pressure was accompanied by small pulse waves in both toes and fingers, owing, presumablly, to decrease in cardiac output. Following administration of ephedrine, the blood pressure rose to or above the initial level, the pulse waves of the toes increased markedly (vasodilatation), while those of the fingers remained small (vasoconstriction). In addition to its other effects, ephedrine acted to restore necessary compensating vasoconstriction to the fingers.

Because vasoconstriction of the fingers seemed necessary for the maintenance of the initial blood pressure during spinal anesthesia, it was considered as an index of vasoconstriction in a larger, but not exactly defined, vascular bed of the upper portion of the body. Similarly, the vasodilatation of the toes was considered as an index of vasodilatation in a large vascular bed of the lower portion of the body, on the basis that vasconstriction of the fingers could hardly be evoked by vasodilatation of the toes alone. The mechanisms for the mediation of the response of compensating 
vasoconstriction were presumed to be none other than those vasomotor reflexes which are in operation normally for the support of blood pressure. The heightened response in the fingers was attributed to the call for increased vasoconstriction wherever possible in the face of loss of ability of the vascular bed of the lower portion of the body to respond.

\section{BIBLIOGRAPHY}

1. Cournand, A., Riley, R. L., Bradley, S. E., Breed, E. S., Noble, R. P., Lauson, H. D., Gregerson, M. I., and Richards, D. W., Jr., Studies of the circulation in clinical shock. Surgery, 1943, 13, 964.

2. Turner, R. H., Studies in the physiology of blood vessels in man. Apparatus and methods. I. A sensitive plethysmophygmograph for a portion of the finger. J. Clin. Invest., 1937, 16, 777.

3. Burch, G. E., Cohn, A. E., and Neumann, C., A study by quantitative methods of the spontaneous variations in volume of the finger tip, toe tip, and postero-superior portion of the pinna of resting normal white adults. Am. J. Physiol., 1942, 136, 433.

4. Neumann, C., Lhamon, W. T., and Cohn, A. E., A study of factors (emotional) responsible for changes in the pattern of spontaneous rhythmic fluctuations in the volume of the vascular bed of the finger tip. J. Clin. Invest., 1944, 23, 1.

5. Johnson, C. A., The digital plethysmograph as a measure of the peripheral circulation. Surg., Gynec., and Obst., 1940, 70, 31.

6. Burch, J. C., Harrison, T. R., and Blalock, A., A comparison of the effects of hemorrhage under ether anesthesia and under spinal anesthesia. Arch. Surg., 1930, 21, 693.

7. Co Tui, F., Spinal anesthesia. The experimental basis for some prevailing clinical practices. Arch. Surg., 1936, 33, 825.

8. Gaddum, J. H., and Kwiatkowski, $H$., The action of ephedrine. J. Physiol., 1938, 94, 87.

9. Starr, I., Gamble, C. J., Margolies, A., Donal, J. S., Jr., Joseph, N., and Eagle, E., A clinical study of the action of 10 commonly used drugs on cardiac output, work and size; on respiration, on metabolic rate and on the electrocardiogram. J. Clin. Invest., 1937, 16, 799.

10. Neumann, C., Sellers, E. A., Rovenstine, E. A., Cohn, A. E., and Rule, C., Influence of spinal and regional anesthesia upon vasoconstriction and vasodilatation of small peripheral blood vessels. Proc. Soc. Exper. Biol. and Med., 1943, 53, 159.

11. Unpublished data. 